\title{
Non-invasive investigations successfully select patients for temporal lobe surgery
}

\author{
Christine Kilpatrick, Mark Cook, Andrew Kaye, Michael Murphy, Zelko Matkovic
}

Department of

Neurology

C Kilpatrick

M Cook

Z Matkovic

Department of Neurosurgery, The Melbourne

Neuroscience Centre, The Royal Melbourne Hospital, Victoria,

Australia

A Kaye

Department of

Neurology,

M Cook

Department of

Neurosurgery, St

Vincent's Hospital,

Melbourne, Victoria,

Australia

M Murphy

Correspondence to: Dr Christine Kilpatrick, The Melbourne Neuroscience Centre, The Royal

Melbourne Hospital, Grattan

Street, Parkville 3050,

Victoria, Australia.

Received 23 August 1996 and in revised form 7 March 1997

Accepted 17 March 1997

\begin{abstract}
Objectives-There is controversy regarding the need for invasive monitoring in the preoperative assessment of patients with temporal lobe epilepsy. The use of a series of non-invasive investigations in identifying the seizure focus is reported in 75 consecutive adults referred for epilepsy surgery.

Methods-All had video-EEG monitoring using scalp electrodes, high resolution MRI, and neuropsychology assessment. Other investigations included volumetric MRI, PET, and ictal and interictal SPECT. The seizure focus was localised and surgery offered if MRI disclosed unilateral hippocampal atrophy or a foreign tissue lesion and other investigations were either concordant or not discordant. Results-In 68 patients the seizure focus was localised and three patients were inoperable. Sixty five patients have been offered surgery and 50 have undergone temporal lobe surgery and have a follow up of at least 12 months (mean 24 months). All had pathology: hippocampal sclerosis 34 , dysembryoblastic neuroepithelial tumour six, cavernoma four, dysplasia two, low grade glioma two, ganglioglioma two. Thirty nine patients $(78 \%)$ are seizure free postoperatively, 29/34 with hippocampal sclerosis and 10/16 with a foreign tissue lesion. Of the 11 patients with postoperative recurrent seizures, eight have a $>\mathbf{9 0} \%$ reduction in seizure frequency and three have $<90 \%$ reduction in seizure frequency but a worthwhile improvement.

Conclusions-Non-invasive investigations successfully select most patients for temporal lobe surgery.
\end{abstract}

(F Neurol Neurosurg Psychiatry 1997;63:327-333)

Keywords: non-invasive investigations; MRI; temporal lobe surgery

Successful surgical treatment of medically refractory partial epilepsy requires accurate localisation of the site of seizure origin. Before recent developments in both structural and functional neuroimaging, localisation of a seizure focus depended almost entirely on electrographic recordings. In many patients scalp EEG recordings were combined with prolonged intracranial monitoring which is labour intensive, expensive, and has potential for significant morbidity. In recent years there has been a shift away from invasive monitoring with more emphasis on the role of neuroimaging in the selection of patients for epilepsy surgery. Brain MRI including volumetric analysis allows identification of a structural cause in most of these patients, ${ }^{12}$ and functional imaging with single photon emission computed tomography (SPECT) ${ }^{3}$ and PET $^{4}$ often provide confirmatory evidence of the seizure focus, localised by other methods. In the light of these advances the indications for invasive EEG monitoring in the selection of patients for temporal lobe surgery have become less clear. We report therefore, the results of the presurgical evaluation and outcome from epilepsy surgery at The Royal Melbourne Hospital and St Vincent's Hospital Comprehensive Epilepsy Programme, where a non-invasive protocol emphasising neuroimaging is used in the assessment and selection of patients for temporal lobe surgery.

\section{Methods}

PATIENT POPULATION

Seventy five consecutive patients with intractable partial epilepsy referred for epilepsy surgery and who completed a presurgical assessment between April 1993 and July 1995, formed the basis of this study. There were 37 males and 38 females, aged from 15-58 (mean (SD), 37 (11)) years. All patients had longstanding epilepsy inadequately controlled by antiepileptic medications, experiencing at least two seizures a month.

PRESURGICAL EVALUATION

A clinical history was obtained and a full neurological examination performed on all patients. Routine interictal scalp EEG recordings (16 channels) were available for review in 65 patients. The presence and location of epileptiform and slow wave activity was determined. All patients underwent continuous 24 hour video-EEG scalp monitoring, using 16 channels according to the standard 10-20 international system. At least three spontaneous habitual seizures were recorded in each patient. In most patients antiepileptic medications were reduced to precipitate seizures. The video-EEG recordings were reviewed by two neurologists (CK, MC). The seizure semiology was assessed in detail noting lateralising clinical features such as unilateral upper limb dystonia, unilateral arm paresis, late head turning, unilateral facial twitching, unilateral limb clonus, and postictal dysphasia. Ictal EEG recordings were reviewed, noting the site and time of onset and pattern of the ictal activity and the presence and site of postictal changes. 
All patients underwent an MRI on a GE 1.5 Tesla scanner. In 55 of the 75 patients the MRI had been performed before admission for presurgical assessment. Volumetric MRI including measurement of hippocampal volumes was carried out on all but one patient, who did not have a foreign tissue lesion. All hippocampal volume measurements were performed by one neurologist (MC) using a method previously reported. ${ }^{5}$ The degree of atrophy of the hippocampus was expressed as a ratio of the smaller to the larger hippocampal volume, measured in $\mathrm{mm}^{3}$; normal ratios being greater than $0.95 .^{5}$ All patients with a foreign tissue lesion had MRI visual analysis of mesial temporal structures.

Interictal (47 patients) and ictal or immediate postictal (20 patients) SPECT and interictal PET (35 patients) scans were used in the localisation and lateralisation of the seizure focus. PET was not performed during 1993 and from mid-1994 was performed on most patients with hippocampal sclerosis. Periictal (ictal or immediate postictal) SPECT was obtained in a minority of patients for logistical reasons.

A full neuropsychological assessment was performed in all patients. General intelligence was assessed using the Weschler adult intelligence scale-revised (WAIS-R). Testing of both verbal and non-verbal memory included performance on the Rey auditory verbal learning task (RAVLT), Austin maze, and Osterreith Rey figure. Neuropsychology assessment was performed to ensure the function of the temporal lobe contralateral to the presumed seizure focus was intact, and the neuropsychology deficits were consistent with the side of the presumed seizure focus. These results were not used as a major criterion for seizure localisation. An intracarotid sodium amytal test was performed if the neurosychological assessment suggested dysfunction of the temporal lobe contralateral to the side of the proposed surgery.

LOCALISATION OF SEIZURE FOCUS

Localisation of the seizure focus was based on the results of all the investigations. The focus was localised and surgery offered to patients in whom the MRI disclosed either a foreign tissue lesion or unilateral hippocampal atrophy confirmed on volumetric analysis, and the ictal EEG and seizure semiology when lateralised were either concordant - that is, indicated that the seizure focus was ipsilateral to the side of the MRI lesion, or predominantly not discordant (most of the seizures were not lateralised contralateral to the side of the MRI lesion). In addition interictal PET and peri-ictal SPECT when available, were either concordant or not discordant with the side of the MRI lesion. Interictal SPECT was not used as a major criterion on which to base seizure localisation or lateralisation, but rather as a baseline for interpretation of peri-ictal (ictal or immediate postictal) SPECT studies.
NEUROSURGERY

All patients with hippocampal sclerosis underwent a tailored anterior temporal lobectomy with en bloc excision of the neocortical structures followed by microsurgical resection of the amygdala and en bloc excision of the hippocampal formation and parahippocampal gyrus. The extent of the neocortical resection depended on the side of the procedure; non-dominant included excision of $4 \mathrm{~cm}$ of the superior temporal gyrus and the middle temporal gyrus, and inferior temporal gyrus to the vein of Labbe or $5-6 \mathrm{cms}$; in dominant lobectomy, the superior temporal gyrus was left intact, the middle temporal gyrus was excised for either $4-5 \mathrm{~cm}$ or to the vein of Labbe, and the inferior temporal was excised for either $4.5-5.5 \mathrm{~cm}$ or to the vein of Labbe. Patients with foreign tissue lesions either had an anterior temporal lobectomy, lesionectomy, or neocorticectomy. All patients with lesions had either lesionectomy or neocorticectomy, except for two patients with a tumour, in whom a more extensive anterior temporal lobectomy was performed, as it was thought that complete excision could be achieved with a lesionectomy.

\section{NEUROPATHOLOGY}

The excised temporal lobe including the mesial structures were fixed in $10 \%$ neutral buffered formalin, sliced in a coronal plane, and embedded in paraffin. The coronal sections were stained with haematoxylin and eosin, Kluver and Barrera luxol fast blue, and cresyl violet counter stain. Where indicated, peroxidaseantiperoxidase immunohistochemical staining for neurons and astrocytes with antibodies against synaptophysin and glial fibrillary acidic protein respectively, was also carried out. The hippocampal subfields and dentate gyrus were examined for neuronal loss and gliosis by visual analysis. Hippocampal sclerosis was defined as greater than $50 \%$ neuron loss in CA1 subfield.

\section{ASSESSMENT OF OUTCOME}

Postoperative follow up entailed regular assessment at least every three months in the epilepsy outpatient clinic. Surgical outcome was classified according to a four point scale, modified from Engel classification ${ }^{7}$ - that is, 1, seizure free (with or without auras); $2,>90 \%$ reduction in seizure frequency; $3,<90 \%$ reduction in seizure frequency but a worthwhile improvement; and 4, no worthwhile improvement in seizure frequency. Antiepileptic drugs were continued postoperatively for at least two years.

\section{STATISTICAL ANALYSES}

Fisher's exact test was used to assess differences in proportion of patients seizure free in each pathological group.

\section{Results}

The seizure focus was localised in 68 of the 75 patients (figure). In two of the 68 patients the MRI showed an unresectable lesions: cortical dysplasia in one and arteriovenous malformation in another. One patient was deemed to be inoperable due to poor neuropsychological function. In the remaining 65 patients surgery 


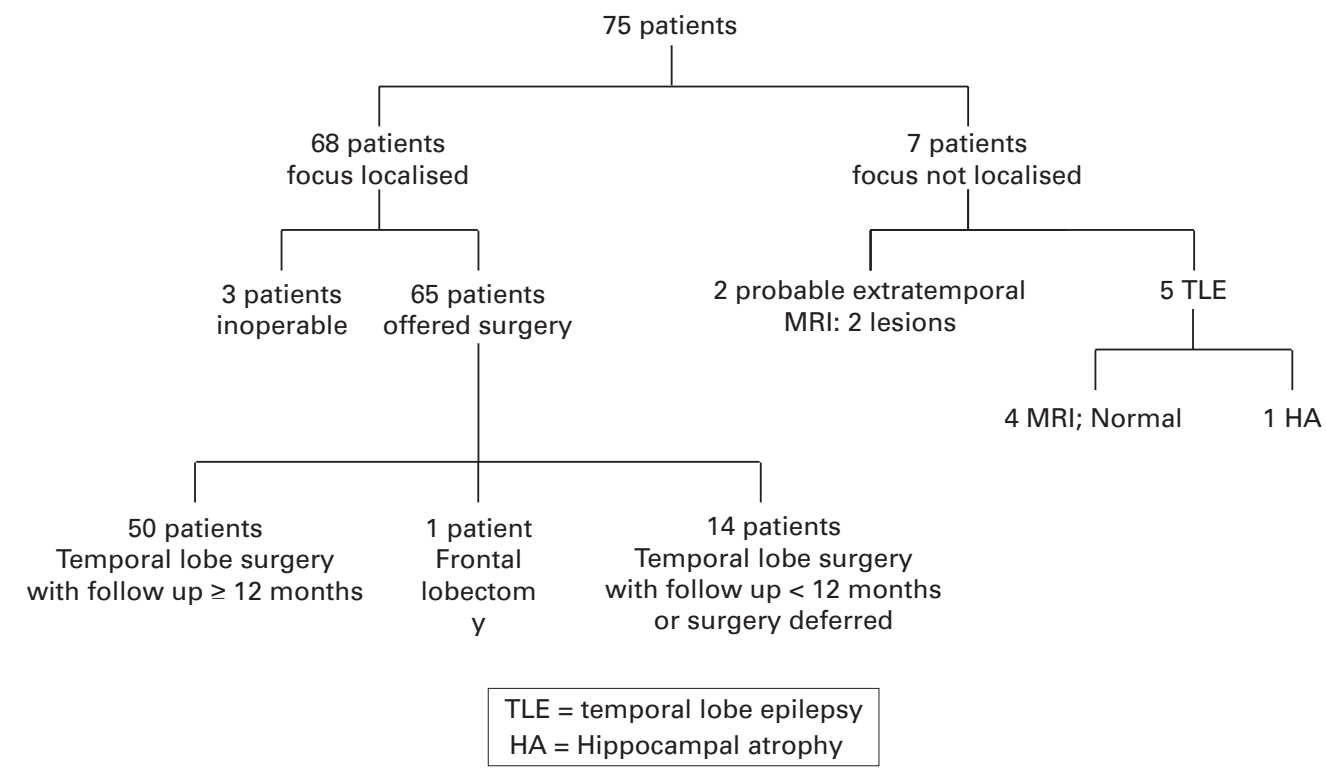

Outcome of seizure localisations in 75 patients referred for epilepsy surgery.

has been offered; 50 have undergone temporal lobe surgery and have a postoperative follow up of at least 12 months. The surgical procedures performed were anterior temporal lobectomy (36), lesionectomy (12), and neocorticectomy (2). One patient was found to have extratemporal seizures and proceeded to a frontal lobectomy. In the remaining 14 patients postoperative follow up is less than 12 months, or surgery has been deferred at the patient's request.

TEMPORAL LOBE SURGERY WITH FOLLOW UP OF AT LEAST 12 MONTHS

Tables 1 and 2 show the results of the investigations and outcome from surgery of the 50 patients who have undergone temporal lobe surgery with a follow up of at least 12 months.

\section{Clinical features}

In the 50 patients who have undergone temporal lobe surgery and have a follow up of at least 12 months, the mean age at onset of habitual seizures was 15 years. Twenty four patients had a history of febrile convulsions of infancy: 20 of 34 patients with hippocampal sclerosis and four of the 16 patients with a foreign tissue lesion.

\section{Interictal EEG}

Interictal EEGs showed lateralised epileptiform or slow wave activity in 25 of the 45 $(56 \%)$ patients and bilateral independent epileptiform activity in one. Lateralised features (epileptiform or slow wave activity) were ipsilateral to the presumed seizure focus in 21 patients and contralateral to the seizure focus in four. In 20 of the 26 patients with abnormal EEGs, epileptiform discharges occurred ipsilateral to the seizure focus in 15 , contralateral in four, and bilateral in one case.

Seizure semiology

One or more lateralised signs concordant with the seizure focus were noted in at least one recorded seizure in $39(78 \%)$ patients, discord- ant lateralised signs in one seizure in one $(2 \%)$ patient, and no lateralising signs were noted in $10(20 \%)$ patients. A motionless stare was the most common initial feature of the complex partial seizure. Oral automatisms were also common $(80 \%)$ and auras were reported in $87 \%$ of patients but were of no lateralising relevance.

\section{Ictal EEG}

Lateralised seizure activity-that is, rhythmic activity $>4 \mathrm{~Hz}$ or $<4 \mathrm{~Hz}$ ipsilateral to the presumed seizure focus was seen in at least one seizure recording in $48(96 \%)$ patients, and in two $(4 \%)$ patients the ictal EEG was consistently non-lateralising. In one patient with hippocampal atrophy, contralateral ictal EEG activity was recorded in one seizure and ipsilateral in two seizures. In $86 \%$ of EEG recordings the ictal activity involving the temporal lobe diffusely began 10-20 seconds after the onset of the clinical seizure. In 18 patients localised postictal slow wave activity ipsilateral to the presumed seizure focus was seen. Postictal slow wave activity contralateral to the presumed seizure focus was not recorded.

\section{$M R I$}

The MRI was abnormal in all patients. Thirty four patients had unilateral hippocampal atrophy: volume ratios ranged from 0.38 indicative of severe atrophy, to 0.88 indicating mild atrophy. In $60 \%$ of the patients, unilateral hippocampal atrophy was detected on visual analysis of the MRI. Sixteen patients had a temporal neocortical foreign tissue lesion. In all except one patient with a foreign tissue lesion, the hippocampal size on visual analysis and when measured volumetrically was normal, with no signal abnormality in mesial temporal structures on visual analysis of coronal T2 weighted images. One patient with cortical dysplasia had an atrophic hippocampus ipsilateral to the neocortical lesion. 
Table 1 Results of investigations and postoperative outcome in 34 patients with hippocampal sclerosis

\begin{tabular}{|c|c|c|c|c|c|c|c|c|c|c|c|c|c|c|}
\hline Sex & Age & $M R I$ & $\begin{array}{l}\text { Hippo } \\
\text { Ratio }\end{array}$ & $\begin{array}{l}E E G \\
\text { I/Ictal }\end{array}$ & $\begin{array}{l}\text { Seizure } \\
\text { Semiology }\end{array}$ & $\begin{array}{l}\text { EEG } \\
\text { Ictal }\end{array}$ & $\begin{array}{l}\text { SPECT } \\
\text { I/Ictal }\end{array}$ & $\begin{array}{l}\text { SPECT } \\
\text { Ictal }\end{array}$ & PET & $\begin{array}{l}\text { Neuro/ } \\
\text { psych }\end{array}$ & Surgery & Pathology & Outcome & $\begin{array}{l}\text { Follow up } \\
\text { (months) }\end{array}$ \\
\hline $\mathrm{F}$ & 55 & LHA & 0.53 & Normal & CLD,CAP & Left & ND & ND & Left & NL & L ATL & HS & 1 & 23 \\
\hline $\mathrm{F}$ & 39 & LHA & 0.56 & Normal & CLD,FC,Dys & Left & $\mathrm{B} / \mathrm{L} \mathrm{T}$ & Left & Left & $\mathrm{L}$ & L ATL & HS & 1 & 26 \\
\hline M & 36 & LHA & 0.51 & Normal & CLD,CAP & Left & $\mathrm{ND}$ & ND & ND & $\mathrm{L}$ & L ATL & HS & 1 & 22 \\
\hline $\mathrm{F}$ & 17 & RHA & 0.62 & Normal & CLD,FC & Right & Right $\mathrm{T}$ & ND & Right & $\mathrm{L}$ & R ATL & HS & 1 & 35 \\
\hline $\mathrm{M}$ & 43 & RHA & 0.64 & Right & $\mathrm{CAP}$ & Right & $\mathrm{ND}$ & ND & ND & NL & R ATL & HS & 1 & 17 \\
\hline $\mathrm{F}$ & 53 & RHA & 0.59 & Right & CAP & Right & ND & ND & ND & NL & R ATL & HS & 2 & 32 \\
\hline$M$ & 44 & LHA & 0.83 & Left & CLD,CAP & Left & Left F & ND & ND & $\mathrm{L}$ & L ATL & HS & 1 & 34 \\
\hline $\mathrm{F}$ & 33 & RHA & 0.62 & Right & CLD & Right & Right $\mathrm{T}$ & ND & ND & $\mathrm{L}$ & R ATL & HS & 1 & 37 \\
\hline M & 47 & LHA & 0.56 & Left & NL & Left & $\mathrm{ND}$ & ND & Left & NL & L ATL & HS & 1 & 24 \\
\hline M & 19 & RHA & 0.77 & Right & CLD & Right & ND & ND & $\mathrm{ND}$ & NL & R ATL & HS & 1 & 22 \\
\hline$M$ & 47 & RHA & 0.75 & Right & CLD & Right & ND & ND & Normal & NL & R ATL & HS & 1 & 13 \\
\hline $\mathrm{F}$ & 26 & RHA & 0.48 & Normal & CAP & Right & Right $\mathrm{T}$ & ND & ND & NL & R ATL & HS & 1 & 18 \\
\hline $\mathrm{F}$ & 31 & RHA & 0.62 & Normal & CAP & Right & Right T & Right & Right & $\mathrm{L}$ & R ATL & HS & 1 & 16 \\
\hline $\mathrm{F}$ & 45 & RHA & 0.48 & Normal & CLD & Right & $\mathrm{ND}$ & $\mathrm{ND}$ & Right & NL, W & R ATL & HS & 1 & 12 \\
\hline$M$ & 40 & LHA & 0.49 & Normal & CLD,CAP & Left & Left T & ND & Left & $\mathrm{L}$ & L ATL & HS & 2 & 15 \\
\hline $\mathrm{F}$ & 24 & LHA & 0.76 & - & CLD & Left & Left $T$ & Left & $\mathrm{ND}$ & $\mathrm{L}$ & L ATL & HS & 1 & 20 \\
\hline M & 23 & RHA & 0.53 & Right & NL & Right & Right $\mathrm{T}$ & ND & Right & $\mathrm{L}$ & R ATL & HS & 1 & 24 \\
\hline$M$ & 40 & RHA & 0.69 & Normal & NL & Right & $\mathrm{ND}$ & ND & Right & $\mathrm{NL}, \mathrm{W}$ & R ATL & HS & 1 & 27 \\
\hline M & 27 & RHA & ND & Normal & CAP & Right & Right $\mathrm{T}$ & Right & ND & $\mathrm{NL}, \mathrm{W}$ & R ATL & HS & 1 & 38 \\
\hline $\mathrm{F}$ & 34 & LHA & 0.64 & Left & CLD,CAP & Left & Normal & $\mathrm{ND}$ & Left & NL & L ATL & HS & 1 & 19 \\
\hline $\mathrm{F}$ & 23 & RHA & 0.66 & Normal & CLD,FC & Right & ND & ND & Right & NL & R ATL & HS & 2 & 17 \\
\hline M & 24 & RHA & 0.60 & Normal & $\mathrm{CAP}, \mathrm{FC}$ & Right & Right T & ND & ND & $\mathrm{L}$ & R ATL & HS & 1 & 26 \\
\hline$M$ & 44 & LHA & 0.63 & Left & CLD,Dys & Left & Left T & Left & Left & $\mathrm{L}$ & L ATL & HS & 1 & 37 \\
\hline M & 50 & LHA & 0.51 & Normal & CLD,FC & Left & ND & ND & Left & NL & L ATL & HS & 2 & 25 \\
\hline $\mathrm{F}$ & 30 & LHA & 0.60 & Normal & ILD & Left & Left $T$ & Left & ND & $\mathrm{L}$ & L ATL & HS & 1 & 22 \\
\hline F & 38 & LHA & 0.69 & Right & NL & Left & ND & ND & Left & $\mathrm{L}, \mathrm{W}$ & L ATL & HS & 1 & 14 \\
\hline $\mathrm{F}$ & 39 & LHA & 0.52 & Left & CLD,CAP & NL & Normal & Left & Left & NL & L ATL & HS & 2 & 28 \\
\hline M & 30 & LHA & 0.66 & - & CLD & Left & Right T & Left & ND & NL & L ATL & HS & 1 & 31 \\
\hline M & 56 & LHA & 0.79 & Left & CLD & Left & Left $T$ & ND & Left & NL & L ATL & HS & 1 & 26 \\
\hline $\mathrm{F}$ & 34 & RHA & 0.54 & Normal & CAP & Left & ND & ND & Normal & $\mathrm{NL}, \mathrm{W}$ & L ATL & HS & 1 & 19 \\
\hline M & 28 & RHA & 0.64 & Normal & CLD,CAP,FC & Right & ND & ND & ND & NL & R ATL & HS & 1 & 18 \\
\hline $\mathrm{F}$ & 40 & RHA & 0.38 & Left & CLD,CAP,FC & Right & Left $\mathrm{T}$ & INC & Right & NL & R ATL & HS & 1 & 24 \\
\hline$M$ & 35 & LHA & 0.77 & Left & NL & Left & Normal & ND & Left & NL & L ATL & HS & 1 & 15 \\
\hline $\mathrm{F}$ & 57 & RHA & 0.88 & Right & CAP & Right & Right $\mathrm{T}$ & ND & Right & $\mathrm{L}$ & R ATL & HS & 1 & 18 \\
\hline
\end{tabular}

$\mathrm{HA}=$ hippocampal atrophy; $\mathrm{ND}=$ not done; $\mathrm{I} / \mathrm{Ictal}=$ interictal; $\mathrm{T}=$ temporal; $\mathrm{CLD}=$ contralateral upper limb dystonia; $\mathrm{B} / \mathrm{L}=\mathrm{bilateral} ; \mathrm{CAP}=$ contralateral arm paresis; $\mathrm{F}=$ frontal; $\mathrm{FC}=$ facial clonus; $\mathrm{W}=\mathrm{Wada}$; Dys=dysphasia; $\mathrm{ATL}=$ anterior temporal lobectomy; ILD=ipsilateral; upper limb dystonia; HS=hippocampal sclerosis; $\mathrm{NL}=$ non-lateralising; INC $=$ inconclusive

Table 2 Results of investigations and postoperative outcome in 16 patients with a foreign tissue lesion

\begin{tabular}{|c|c|c|c|c|c|c|c|c|c|c|c|c|c|c|}
\hline Sex & Age & $M R I$ & $\begin{array}{l}\text { Hippo } \\
\text { Ratio }\end{array}$ & $\begin{array}{l}\text { EEG } \\
\text { I/Ictal }\end{array}$ & $\begin{array}{l}\text { Seizure } \\
\text { semiology }\end{array}$ & $\begin{array}{l}\text { EEG } \\
\text { Ictal }\end{array}$ & $\begin{array}{l}\text { SPECT } \\
\text { I/Ictal }\end{array}$ & $\begin{array}{l}\text { SPECT } \\
\text { Ictal }\end{array}$ & PET & $\begin{array}{l}\text { Neuro/ } \\
\text { psych }\end{array}$ & Surgery & Pathology & Outcome & $\begin{array}{l}\text { Follow up } \\
\text { (months) }\end{array}$ \\
\hline $\mathrm{F}$ & 38 & RFTL & ND & Right & CLD,CAP & Right & ND & ND & ND & NL & R Lesion & DNET & 1 & 30 \\
\hline $\mathrm{M}$ & 50 & RFTL & ND & Left & NL & Right & ND & ND & ND & $\mathrm{L}$ & R Lesion & Cav & 1 & 15 \\
\hline$M$ & 30 & RFTL & 0.95 & Right & FC & Right & Right T & ND & Right & NL & R Lesion & DNET & 1 & 18 \\
\hline $\mathrm{F}$ & 16 & LFTL & 0.79 & $\mathrm{~B} \backslash \mathrm{L}$ & CLD,CAP,FC & Left & Left $T$ & Left & Left & $\mathrm{L}$ & LNeocort & Dysp & 1 & 24 \\
\hline M & 50 & RFTL & ND & Left & CLD,CHT & Right & ND & ND & ND & $\mathrm{L}$ & R Lesion & DNET & 1 & 18 \\
\hline M & 50 & LFTL & 0.96 & Left & CLD & Left & Normal & Left & ND & NL & L Lesion & DNET & 1 & 36 \\
\hline$M$ & 20 & LFTL & ND & Normal & NL & Left & Left T & Left & ND & $\mathrm{L}$ & L ATL & Glioma & 3 & 34 \\
\hline M & 36 & LFTL & ND & - & CLD & Left & Left $\mathrm{T}$ & Left & ND & $\mathrm{L}$ & L Lesion & Cav & 1 & 36 \\
\hline$M$ & 20 & LFTL & ND & Left & CLD & Left & Left T & ND & ND & $\mathrm{L}$ & L Neocort & Dysp & 2 & 30 \\
\hline M & 45 & LFTL & 0.96 & Normal & NL & NL & Normal & ND & $\mathrm{ND}$ & NL & L Lesion & Cav & 1 & 25 \\
\hline M & 58 & LFTL & ND & Normal & CAP,FC,CHT & Left & Left $\mathrm{T}$ & ND & ND & $\mathrm{L}$ & L Lesion & G/Glioma & 3 & 29 \\
\hline $\mathrm{F}$ & 36 & LFTL & ND & Left & NL & Left & ND & ND & ND & $\mathrm{L}$ & L ATL & G/Glioma & 3 & 24 \\
\hline F & 55 & RFTL & ND & - & CAP & Right & ND & ND & ND & $\mathrm{L}$ & R Lesion & DNET & 1 & 35 \\
\hline$M$ & 44 & LFTL & ND & Left & NL & Left & ND & ND & ND & $\mathrm{L}$ & L Lesion & Glioma & 2 & 20 \\
\hline $\mathrm{F}$ & 18 & LFTL & ND & - & CLD & Left & ND & ND & ND & $\mathrm{L}$ & L Lesion & DNET & 1 & 31 \\
\hline$M$ & 41 & RFTL & 0.95 & Right & CLD & Right & Right $T$ & Right & Right & NL & R Lesion & Cav & 2 & 18 \\
\hline
\end{tabular}

$\mathrm{FTL}=$ foreign tissue lesion; $\mathrm{NL}=$ non-lateralising; $\mathrm{ND}=$ not done; Lesion=lesionectomy; $\mathrm{CLD}=$ contralateral upper limb dystonia; Neocort=neocorticectomy; $\mathrm{CAP}=$ contralateral arm paresis; $\mathrm{ATL}=$ anterior temporal lobectomy; $\mathrm{FC}=$ facial clonus; $\mathrm{DNET}=$ dysembryoblastic neuroepithelial tumour; $\mathrm{CHT}=$ contralateral head turn; Cav=cavernoma; I/Ictal=interictal; Dysp=dysplasia; $\mathrm{T}=$ temporal; $\mathrm{G} / \mathrm{Glioma}=$ ganglioglioma.

SPECT and PET

Ictal or immediate postictal SPECT showed concordant lateralised changes in 12 of 13 $(92 \%)$ and was inconclusive in one patient. Interictal SPECT disclosed temporal hypoperfusion ipsilateral to the seizure focus in 20 of 29 $(69 \%)$, contralateral in two patients, bilateral changes in one, hypoperfusion concordant for lateralisation but not localisation in one case and normal in five cases. PET was performed in 24 patients: 22 with hippocampal atrophy and two with foreign tissue lesions. Hypometabolism of the ipsilateral temporal lobe was present in 22 patients, $20 / 22$ with hippocampal atrophy, and $2 / 2$ with foreign tissue lesions. In two patients with hippocampal atrophy the PET was normal.
NEUROPSYCHOLOGY ASSESSMENT

Neuropsychology testing disclosed that all patients were of at least average intellect. Deficits in verbal or non-verbal memory function consistent with the side of the seizure focus were present in 19 patients and contralateral in six. Six patients proceeded to an intracarotid sodium amytal test ipsilateral to the side of the proposed surgery; the contralateral temporal lobe adequately sustained memory in all.

\section{PATHOLOGY}

Pathological abnormalities were found in all temporal resections: hippocampal sclerosis (34), cavernoma (four), dysembryoblastic neuroepithelial tumour (DNET) (six), dysplasia (two), low grade glioma (two), and ganglio- 
glioma (two). There were no cases of dual pathology.

OUTCOME

The duration of follow up ranged from 12 months to 38 with a mean of 24 months. Thirty nine $(78 \%)$ patients are seizure free (with or without auras). Two of the 39 patients have had occasional auras but no seizures. Eleven patients have ongoing seizures, eight patients have a $>90 \%$ reduction in seizure frequency, and three patients have a less than $90 \%$ reduction in seizure frequency but a worthwhile improvement. Of the 34 patients with hippocampal sclerosis, $29(85 \%)$ are seizure free, whereas 10 of the $16(63 \%)$ patients with a foreign tissue lesion are seizure free $(\mathrm{P}=0.21)$. Of the six patients with a foreign tissue lesion and ongoing seizures, five had an incomplete resection based on surgical, pathological, and MRI findings. Of the five patients with hippocampal sclerosis and postoperative seizures, in two cases seizures occurred after withdrawal of medication, in one case two years postsurgery and in the other in the first few weeks following surgery when medications were inadvertently reduced. This patient has been seizure free for 15 months after reintroduction of treatment. In the other three patients seizures recurred within weeks of surgery and have continued intermittently. In two of these three patients, the ictal EEG and PET were lateralised, concordant with the seizure focus, and in one patient the ictal EEG was consistently not lateralised.

Of the 40 patients with at least 18 months of follow up, $31(78 \%)$ are seizure free with or without auras, 23 of 26 (88\%) with hippocampal sclerosis and eight of $14(57 \%)$ with a foreign tissue lesion $(\mathrm{P}=0.065)$.

MORBIDITY

Postoperative complications occurred in eight $(16 \%)$; all were transient and relatively minor. Three patients developed mild and transient dysphasia (all resolved within two months) after dominant hemisphere anterior temporal lobectomy for hippocampal sclerosis. One patient developed transient diplopia (resolved within three weeks) and one a transient fourth nerve palsy (resolved within four months). Three patients developed a depressive illness which resolved spontaneously.

PATIENTS IN WHOM SEIZURE FOCUS WAS NOT LOCALISED

In seven of the 75 patients $(9 \%)$, the seizure focus was not localised using these noninvasive investigations (figure ${ }^{1}$ ). Of these seven patients, two had probable extratemporal seizures and five temporal seizures. In the two patients with probable extratemporal seizures, the MRI in each case disclosed two lesions, one in the temporal lobe and the other in the ipsilateral frontal lobe, and based on the noninvasive investigations, a decision as to which lesion was responsible for the seizures could not be made. Of the five patients with temporal lobe seizures, in four the MRI on visual analysis and volumetric measurement was normal and the ictal (SPECT and EEG) studies were either non-lateralising or discordant. In one patient the MRI showed mild hippocampal atrophy (ratio 0.89), but results of other investigations were discordant or non-lateralising; the ictal EEG was non-lateralising, postictal SPECT discordant to the MRI finding, and PET normal.

\section{Discussion}

Relatively few studies have reported results of epilepsy surgery using a non-invasive protocol for identifying the seizure focus, ${ }^{8-10}$ even though many recent studies have highlighted the importance of neuroimaging, both structural and functional, in the identification of a seizure focus in patients with a partial epilepsy, and a corresponding reduction in the need for invasive monitoring in the presurgical assessment ${ }^{411-14}$ This study reports successful localisation of the seizure focus $(91 \%)$ and successful outcome from temporal lobe surgery ( $78 \%$ seizure free) in 75 consecutive patients selected for surgery based on non-invasive investigations, using the MRI detected lesion as a major criterion for seizure localisation. We conclude that in most patients the seizure focus can be accurately localised using non-invasive investigations, including high resolution MRI.

Sperling and colleagues reported a noninvasive protocol based on EEG findings, both ictal and interictal with relatively little emphasis on MRI findings. ${ }^{8}$ Eighty per cent of patients who proceeded to surgery were seizure free, but in only $50 \%$ of patients assessed could the seizure focus be identified using this protocol. In a recent study of 22 patients without temporal lobe foreign tissue lesions, $82 \%$ were seizure free using a non-invasive protocol which included visual analysis of the MRI, but not volumetric assessment. ${ }^{10}$ These patients, however, represented only about $20 \%$ of all patients at that centre proceeding to surgery.

It could be argued that in this study there is a bias in referral, given that 71 of 75 (95\%) patients had an MRI lesion. Although patients with a lesion on MRI are more likely to be referred for presurgical assessment, a bias common to most surgical series, of note $27 \%$ of patients had not had an MRI before admission for presurgical assessment and most had not had volumetric analysis and in many cases based on visual analysis, the MRI was thought to be normal. No patient was denied assessment because the MRI was normal. After MRI volumetric assessment the MRI was normal in only four patients.

In support of our approach of using the MRI lesion as the major criterion for selection for surgery, recent studies report the value of MRI using both visual and volumetric analysis, in predicting a good surgical outcome. Garcia et al reported a better outcome in patients with unilateral hippocampal atrophy detected on visual analysis of the MRI ( $96 \%$ seizure free), than patients with a normal scan $(50 \%$ seizure free). ${ }^{11}$ Jack et al reported the best outcome in those patients with hippocampal atrophy detected on volumetric assessment of the MRI. ${ }^{13}$ Berkovic et al reported a better postoperative 
outcome in patients with an MRI lesion, either unilateral hippocampal atrophy or a foreign tissue lesion, compared with patients with a normal MRI. ${ }^{12}$ Similar results have been reported by others. ${ }^{15-17}$

Although the present study placed considerable emphasis on the MRI lesion in establishing the seizure focus, the results of the ictal EEG recordings and seizure semiology were also major criteria in the preoperative assessment of the seizure focus. Consistent with the findings of Williamson et al who assessed the ictal scalp EEG findings in patients with mesial temporal lobe seizures confirmed by depth electrode recording with an excellent surgical outcome ${ }^{18}$ concordant lateralised ictal EEG seizure activity was recorded in most patients although usually not until after the onset of the clinical seizure, and concordant postictal slowing was a common useful localising and lateralising feature.

Several studies have assessed the role of interictal EEG findings, both surface and intracranial, in the localisation of the seizure focus and influence on the postoperative outcome. The results, however, have been inconsistent with some reporting a good correlation between concordant unilateral interictal epileptiform activity and surgical outcome, ${ }^{19}{ }^{20}$ with others reporting contralateral interictal epileptiform activity as not being a negative prognostic feature. ${ }^{21}{ }^{22}$ The results of this study are difficult to compare with these reports as these were usually based on prolonged interictal recordings, some of which used intracranial recordings. In the present study however, the finding of contralateral interictal epileptiform activity in four patients was not a negative prognostic feature as all four patients are seizure free.

The results of surgery for epilepsy reported in this study are comparable with other series including those in which intracranial monitoring was commonly used. ${ }^{71012192023}$ The follow up however is relatively brief, and hence it is necessary to be cautious as to the long term prognosis of these patients. Berkovic et al found the percentage of patients achieving a two year seizure free period after temporal lobectomy changes with time, and that patients with hippocampal sclerosis are at particular risk of late recurrence of seizures. ${ }^{12}$ Given that all patients in the present study had pathology in excised tissue, and pathology known to be epileptogenic, it is reasonable to accept that the seizure focus was correctly localised in most cases. In support of this is the finding that patients with normal excised tissue have a poorer outcome than those with pathology..$^{101224}$

In the current study the outcome in patients with foreign tissue lesions ( $63 \%$ seizure free) was not as good as those with hippocampal sclerosis ( $85 \%$ seizure free). This finding is contrary to other studies which report a greater percentage of patients with tumour or other foreign tissue lesion achieving a seizure free status than those without a foreign tissue lesion, most of whom had hippocampal sclerosis. ${ }^{812}$ Our findings are in part due to the fact that in all but one patient with a foreign tissue lesion and ongoing seizures, complete excision of the lesion could not be achieved. In addition, in our series there were no cases of normal pathology, whereas in other series a significant proportion of patients without a foreign tissue lesion had normal pathology, which would in part account for the relatively poorer outcome in this non-lesional group. ${ }^{8}{ }^{12}$

Five of the 34 patients with hippocampal sclerosis are not seizure free. The reason for the poor outcome in these patients is unclear. Two patients had seizures after drug withdrawal. One patient who has ongoing seizures but with greater than $90 \%$ reduction in seizure frequency, had non-lateralised ictal EEG but concordant lateralised PET and definite hippocampal sclerosis pathologically. Using the protocol that invasive monitoring is indicated if seizure onset on scalp EEG is either nonlateralised or bitemporal, then this latter patient would have proceeded to invasive investigations and these results may have suggested a poorer outcome. ${ }^{20}$ In the other two patients, results of non-investigations were concordant with the MRI lesion. These patients have not been restudied to assess the origin of their recurrent seizures and it is possible that these seizures are originating from the non-operated temporal lobe and that preoperative intracranial monitoring would have detected bitemporal foci or possibly seizures from the opposite temporal lobe. Of the six patients with a foreign tissue lesion and ongoing seizures, all but one had an incomplete excision, the other had a cavernoma which may not have been microscopically fully excised.

The aim of this report is not to undermine the value and place of invasive monitoring in the presurgical assessment, but rather to emphasise that in many patients the seizure focus can be accurately localised using noninvasive investigations - in particular, structural neuroimaging with high resolution MRI. Video-EEG monitoring is essential to confirm the diagnosis of complex partial seizures of temporal lobe origin, and to ensure that ictal EEG onset is not discordant. PET and SPECT and neuropsychological assessment provide further evidence of the site of the seizure focus. Clearly, even if all tests of the seizure focus including an MRI lesion are concordant, some patients will not have a good outcome and the reasons for this remain unclear. Bilateral asymmetric hippocampal sclerosis is one explanation.

Although many studies have assessed the value of a particular imaging technique or compared one with another in the ability to localise a seizure focus, or assessed the predictive value of scalp EEG localisation of seizure onset with respect to postoperative outcome, studies have not compared combinations of non-invasive investigations including MRI in the ability to accurately localise the seizure focus. Such studies are needed to better understand the role of the various imaging and noninvasive EEG techniques in the assessment of patients before surgery and to further define 
the role of intracranial EEG monitoring in the management of these patients.

1 Jackson GD. New techniques in magnetic resonance and epilepsy. Epilepsia 1994;35(suppl 6):S2-13.

2 Spencer SS. MRI and epilepsy surgery [editorial]. Neurology 1995; $45: 1248-50$

3 Berkovic SF, Newton MR, Chiron C, Dulac O. Single photon emission computed tomography. In: Engel J Jr, ed. Sur gical treatment of the epilepsies. New York: Raven Press, 1993:233-43.

4 Theodore WH, Sato S, Kufta C, Balish MB, Bromfield EB Leiderman DB. Temporal lobectomy for uncontrolled seizures: the role of positron emission tomography. Ann Neurol 1992;32:789-94.

5 Cook MJ, Fish DR, Shorvon SD, Straughan K, Stevens JM. Hippocampal volumetric and morphometric studies in frontal and temporal lobe epilepsy. Brain 1992;115:100115.

6 Cook MJ. Mesial temporal sclerosis and volumetric investigations. Acta Neurol Scand Suppl 1994;152:109-15.

7 Engel J Jr, Van Ness PC, Rasmussen TB, Ojemann LM Outcome with respect to seizures. In: Engel J Jr, ed. SurgiOutcome with respect to seizures. In: Engel J Jr, ed. Surgi-
cal treatment of the epilepsies. 2nd ed. New York: Raven Press cal treatment of th

8 Sperling MR, O'Connor MJ, Saykin AJ, Phillips CA, Morrell MJ, Bridgman PA, et al. A non-invasive protoco for anterior temporal lobectomy. Neurology 1992;42:41622.

9 Murro AM, Park YD, King DW, Gallagher BB, Smith JR, et al. Seizure localization in temporal lobe epilepsy: comparison of scalp sphenoidal EEG and volumetric MRI Neurology 1993;43:2531-3.

10 Thadani VM, Williams PD, Berger R, Spencer SS, Spencer intracranial EEG recording: criteria for patient selection. Epilepsia 1995;36:7-15.

11 Garcia PA, Laxer KD, Barbaro NM, Dillon WP. Prognostic value of qualitative magnetic resonance imaging hippocampal abnormalities in patients undergoing temporal lobectomy for medically refractory seizures. Epilepsia 1994;35: 520-4.

12 Berkovic SF, MacIntosh AM, Kalnins RM, Jackson GD, Fabinyi GCA, Brazenor GA, et al. Pre-operative MRI pre-
dicts outcome of temporal lobectomy: an actuarial analysis. dicts outcome of temporal lo
Neurology $1995 ; 45: 1358-63$.
13 Jack CR Jr, Sharbrough FW, Cascino GD, Hirschorn KA, O'Brien PC, Marsh WR. Magnetic resonance image-based hippocampal volumetry: correlation with outcome after temporal lobectomy. Ann Neurol 1992;31:138-46.

14 Jack CR Jr, Mullar BP, Sharbrough FW, Cascino GD, Hauser MF, Krecke KN, et al. Intractable non-lesional epilepsy of temporal lobe origin: lateralization by interictal SPECT versus MRI. Neurology 1994;44:829-36.

15 Kuzniecky R, Burgard S, Faught E, Morawetz R, Bartolucci A. Predictive value of magnetic resonance maging in temporal lobe epilepsy surgery. Arch Neurol 1993;50:65-9.

16 Heinz R, Ferris N, Lee EK, Radtke R, Crain B, Hoffman $\mathrm{JM}$, et al. MRI and positron emission tomography in the diagnosis of surgically correctable temporal lobe epilepsy. AFNR Am f Neuroradiol 1994;15:1341-8.

17 Oliver B, Russi A. What is needed for resective epilepsy surgery from a neurosurgical point of view. Acta Neurol Scand Suppl 1994;152:187-9.

18 Williamson PD, French JA, Thadani VM, Kim JH, Novelly RA, Spencer SS, et al. Characteristics of medial temporal lobe epilepsy: II. Interictal and ictal scalp electroencephalography, neuropsychological testing, neuroimaging, surgical results, and pathology. Ann Neurol 1993;34:781-7.

19 Chung MY, Walczak TS, Lewis DV, et al. Temporal lobectomy and independent bitemporal interictal activity: what degree of lateralization is sufficient? Epilepsia 1991;32:195-201.

20 Hufnagel A, Elgar CE, Pelo H, Zentner J, Wolf HK, Schramm J, Wiestler OD. Prognostic significance of ictal and interictal epileptiform activity in temporal lobe epilepsy. Epilepsia 1994;35:1146-53.

21 Engel J, Driver MV, Falconer MA. Electrophysiological correlates of pathology and surgical results in temporal lobe epilepsy. Brain 1975;98:129-56.

22 Lieb JP, Engel J, Gevins A, Crandall P. Surface and deep EEG correlates of surgical outcome in temporal lobe epilepsy. Epilepsia 1981;22:515-38.

23 Primrose DC, Ojemann GA. Outcome of resective surgery for temporal lobe epilepsy. In: Luders H, ed. Epilepsy surgery. New York: Raven Press, 1992:601-9.

24 Falconer MA. Mesial temporal (Ammon's) sclerosis as a common cause of epilepsy: aetiology, treatment, and prevention. Lancet 1974;ii:767-70. 